\title{
Forward elastic scattering and Pomeron models
}

\author{
M. Broilo, ${ }^{1, *}$ E. G. S. Luna, ${ }^{1, \dagger}$ and M. J. Menon ${ }^{2, \dagger}$ \\ ${ }^{1}$ Instituto de Física, Universidade Federal do Rio Grande do Sul, \\ CEP 91501-970, Porto Alegre - RS, Brazil \\ ${ }^{2}$ Instituto de Física Gleb Wataghin, Universidade Estadual de Campinas, \\ CEP 13083-859, Campinas - SP, Brazil
}

(Received 26 July 2018; published 5 October 2018)

\begin{abstract}
Recent data from LHC13 by the TOTEM Collaboration indicate an unexpected decrease in the value of the $\rho$ parameter and a $\sigma_{\text {tot }}$ value in agreement with the trend of previous measurements at 7 and $8 \mathrm{TeV}$. These data at $13 \mathrm{TeV}$ are not simultaneously described by the predictions from Pomeron models selected by the COMPETE Collaboration but show agreement with the maximal Odderon dominance, as recently demonstrated by Martynov and Nicolescu. Here, we present a detailed analysis on the applicability of Pomeron dominance by means of a general class of forward scattering amplitude, consisting of even-undercrossing leading contributions associated with single, double, and triple poles in the complex angular momentum plane and subleading even and odd Regge contributions. The analytic connection between $\sigma_{\text {tot }}$ and $\rho$ is obtained by means of singly subtracted dispersion relations, and we carry out fits to $p p$ and $\bar{p} p$ data in the interval $5 \mathrm{GeV}-13 \mathrm{TeV}$. The data set comprises all the accelerator data below $7 \mathrm{TeV}$, and we consider two independent ensembles by adding either only the TOTEM data or the TOTEM and ATLAS data at the LHC energy region. In the data reductions to each ensemble, the uncertainty regions are evaluated with both one and two standard deviations ( $\sim 68 \%$ and $\sim 95 \%$ CL, respectively). Besides the general analytic model, we investigate four particular cases of interest, three of them typical of outstanding models in the literature. We conclude that, within the experimental and theoretical uncertainties and both ensembles, the general model and three particular cases are not able to describe the $\sigma_{\text {tot }}$ and $\rho$ data at $13 \mathrm{TeV}$ simultaneously. However, if the discrepancies between the TOTEM and ATLAS data are not resolved, one Pomeron model, associated with double and triple poles and with only 7 free parameters, seems not to be excluded by the complete set of experimental information presently available.
\end{abstract}

DOI: 10.1103/PhysRevD.98.074006

\section{INTRODUCTION}

In elastic hadron-hadron collisions, the forward scattering is characterized by two physical observables - the total cross section, $\sigma_{\text {tot }}$, and the $\rho$ parameter. In terms of the scattering amplitude $\mathcal{A}$ and its Mandelstam variables ( $s$ and $t$, energy and momentum transfer squared in the c.m. system), the former is given by the optical theorem, which at high energies can be expressed by [1]

$$
\sigma_{\text {tot }}(s)=\frac{\operatorname{Im} \mathcal{A}(s, t=0)}{s},
$$

\footnotetext{
*mateus.broilo@ufrgs.br

†luna@if.ufrgs.br

”menon@ifi.unicamp.br
}

Published by the American Physical Society under the terms of the Creative Commons Attribution 4.0 International license. Further distribution of this work must maintain attribution to the author(s) and the published article's title, journal citation, and DOI. Funded by SCOAP ${ }^{3}$. and the latter, associated with the phase of the amplitude, is defined by

$$
\rho(s)=\frac{\operatorname{Re} \mathcal{A}(s, t=0)}{\operatorname{Im} \mathcal{A}(s, t=0)},
$$

where $t=0$ indicates the forward direction.

Since the real and imaginary parts of the amplitude can be formally correlated by means of dispersion relations, Eqs. (1) and (2) provide a fundamental physical connection between the phase of the amplitude $(\rho)$ and the total probability of the hadronic interaction $\left(\sigma_{\text {tot }}\right)$, as a function of the energy. However, despite their rather simple analytic forms, the investigation of these two quantities in terms of the energy, constitute a long-standing challenge in the study of the hadronic interactions [2].

In the experimental context, to access the forward and near forward region demands complex and sophisticated instrumentation and data analyses. In addition, the difficulties grow progressively as the energy increases. For example, the $\rho$ parameter is determined in the region of 
interference between the Coulomb and hadronic interactions, which are of the same magnitude at values of the momentum transfer proportional to the inverse of the total cross section (see, for example, [3], Sec. IV). As a consequence of the rise of $\sigma_{\text {tot }}$ at the highest energies, it becomes extremely difficult to reach this region as the energy increases.

In the theoretical QCD context, this deep (extreme) infrared region $(t \rightarrow 0)$ is not expected to be accessed by perturbative techniques. A crucial point concerns the absence of a nonperturbative approach able to predict the energy dependence of $\sigma_{\text {tot }}$ and $\rho$ from first principles and without model assumptions.

In the phenomenological context, beyond classes of models including other physical quantities, ${ }^{1} \sigma_{\text {tot }}(s)$ and $\rho(s)$ are usually investigated by means of amplitude analyses, an approach based on the Regge-Gribov theory and analytic $S$-Matrix concepts. In this formalism [5,6], the singularities in the complex angular momentum $J$-plane ( $t$ channel) are associated with the asymptotic behavior of the elastic scattering amplitude in terms of the energy ( $s$ channel). In the general case, associated with a pole of $\operatorname{order} N$, the contribution to the imaginary part of the forward amplitude in the $s$ channel is $s^{\alpha_{0}} \ln ^{N-1}(s)$, where $\alpha_{0}$ is the intercept of the trajectory (see Appendix B in [7] for a recent short review). Therefore, for the total cross section, we have

$$
\sigma_{\mathrm{tot}}(s) \propto s^{\alpha_{0}-1} \ln ^{N-1} s,
$$

and the following possibilities connecting the singularities $(J$-plane) and the asymptotic behavior:

(i) simple pole $(N=1)$ at $J=\alpha_{0}$, with $\alpha_{0}=1 \Rightarrow \sigma$ constant;

(ii) simple pole $(N=1)$ at $J=\alpha_{0} \Rightarrow \sigma \propto s^{\alpha_{0}-1}$;

(iii) double pole $(N=2)$ at $J=\alpha_{0}$, with $\alpha_{0}=1 \Rightarrow$ $\sigma \propto \ln (s)$

(iv) triple pole $(N=3)$ at $J=\alpha_{0}$, with $\alpha_{0}=1 \Rightarrow$ $\sigma \propto \ln ^{2}(s)$.

For an elastic particle-particle and antiparticle-particle scattering, given the above inputs for $\sigma_{\text {tot }}(s)$, through Eq. (1), the even and odd contributions associated with $\operatorname{Im} \mathcal{A}(s, t=0) / s$ are defined (crossing) and the corresponding real parts are obtained by means of dispersion relations (analyticity), leading to $\rho(s)$ in Eq. (2).

Historically, the leading contribution to $\sigma_{\text {tot }}$ at the highest energies has been associated with an even-under-crossing object named Pomeron (from a QCD viewpoint, a color singlet made up of two gluons in the simplest configuration) [6]. Typical Pomeron models consider contributions associated with either a simple pole at $J=\alpha_{0}$ (for example, Donnachie and Landshoff [8] and some QCD-inspired models [9]) or a triple pole at $J=1$ (as selected in the detailed analysis by the COMPETE Collaboration $[10,11]$

\footnotetext{
${ }^{1}$ For recent reviews, see, for example, [2-4].
}

and used in the successive editions of the Review of Particle Physics, by the Particle Data Group (PDG) [12]).

However, recently, new experimental information on $\sigma_{\text {tot }}$ and $\rho$ from LHC13 were reported by the TOTEM Collaboration [13,14]:

$$
\begin{aligned}
\sigma_{\text {tot }} & =110.6 \pm 3.4 \mathrm{mb}, \\
\rho & =0.10 \pm 0.01 \quad \text { and } \\
\rho & =0.09 \pm 0.01,
\end{aligned}
$$

indicating an unexpected decrease in the value of the $\rho$ parameter and $\sigma_{\text {tot }}$ in agreement with the trend of previous measurements at 7 and $8 \mathrm{TeV}$. Indeed, recent investigation concerning bounds on the rise of $\sigma_{\text {tot }}(s)$, including all TOTEM data at 7 and $8 \mathrm{TeV}$ and the $\mathrm{L} \gamma$ parametrization [15], has predicated at $13 \mathrm{TeV}$ the value $\sigma_{\text {tot }}=110.7 \pm 1.2 \mathrm{mb}$, which is in full agreement with the above measurement. However, for $\rho$ at $13 \mathrm{TeV}$ the extrapolation yielded $0.1417 \pm 0.0047$, indicating complete disagreement with the data and far above the experimental result (see Table 4 in [15]). Moreover, the results (3) are not simultaneously described by all the predictions of the Pomeron models from the detailed analysis by the COMPETE Collaboration in 2002 [10], as pedagogically shown in Fig. 18 of [14]).

Remarkably, the odd-under-crossing asymptotic contribution, introduced by Lukaszuk and Nicolescu [16] and named Odderon [17] (from a QCD viewpoint, a colorsinglet made up of three gluons in the simplest configuration) [18], provides quite good descriptions of the experimental data, as predicted by the Avila-GauronNicolescu model [19] and demonstrated recently in the analyses by Martynov and Nicolescu [20,21].

On the other hand, also recently, the above data at $13 \mathrm{TeV}$ have been analyzed by Khoze, Martin, and Ryskin in the context of a QCD-based multichannel eikonal model (Pomeron dominance), tuned in 2013 with data up to $7 \mathrm{TeV}$ [22]. The analysis indicates that the data at $13 \mathrm{TeV}$ are reasonably described without an odd-signature term [23]. Moreover, the authors also argument that the maximal Odderon is inconsistent with the black-disk limit [24]. Very recently, subsequent articles have also discussed possible effects related to Odderon contributions in different contexts [25].

In view of all these recent information and the fact that forward amplitude analyses have favored the Pomeron dominance, at least, up to $8 \mathrm{TeV}$, it seems important to develop detailed tests on the applicability of the Pomeron models by means of a general class of forward scattering amplitudes. With that in mind, we have already reported two forward analyses with Pomeron dominance and including, for the first time, the TOTEM data at $13 \mathrm{TeV}$. In the first work, two models have been tested, without taking into account the uncertainty regions in the data 
reductions [26] (as in the Martynov and Nicolescu analyses $[20,21])$. We concluded that the models are not able to satisfactorily describe the $\sigma_{\text {tot }}$ and $\rho$ data at $13 \mathrm{TeV}$. In the second analysis, we have considered one Pomeron model with six free parameters and have evaluated the uncertainty regions with confidence level (CL) of $90 \%$. We have concluded that the model seems not to be excluded by the bulk of experimental data presently available [27].

In this paper, we shall extend our investigation in several important aspects. The main point is to consider classes of even leading contributions by incorporating different components of several models and investigating the effect of several combinations, with focus on the uncertainties involved in the data reductions. To this end, we shall treat a general parametrization for $\sigma_{\text {tot }}(s)$ consisting of constant, power, logarithmic and logarithmic squared functions of the energy, together with even and odd Reggeons $\left(a_{2} / f_{2}\right.$ and $\rho / \omega$ trajectories, respectively) for the low energy region. The analytic connection with $\rho(s)$ is obtained by means of even and odd singly subtracted dispersion relations. We carry out fits to $p p$ and $\bar{p} p$ data on $\sigma_{\text {tot }}$ and $\rho$ in the interval $5 \mathrm{GeV}-13 \mathrm{TeV}$ through the general parametrization as well as four particular cases, three of them typical of outstanding models in the literature.

However, there is an intrinsic difficulty with this kind of analysis deserving attention from the beginning. In what concerns the $\sigma_{\text {tot }}$ and $\rho$ data, despite the great expectations with the LHC, the experimental information presently available are characterized by discrepancies between the measurements of $\sigma_{\text {tot }}$ by the TOTEM Collaboration and by the ATLAS Collaboration at $7 \mathrm{TeV}$ and mainly at $8 \mathrm{TeV}$. Some consequences of these discrepancies have already been discussed by Fagundes, Menon, and Silva [15] and by us in [27]. In their first analysis, Martynov and Nicolescu present arguments for not including the ATLAS data [20], which, however, have been included in their second analysis [21]. We shall return to these important topics along the paper.

It should be also noted that the uncertainties in the TOTEM measurements of $\sigma_{\text {tot }}$ are essentially systematic (uniform distribution) and not statistical (Gaussian distribution). This fact puts limitations in a strict interpretation of the $\chi^{2}$ test of goodness-of-fit. This point is discussed in certain detail in Ref. [7], Table 1 and Appendix A, especially Sec. A.1.

Here, to address the above question and as in previous analyses [7,15,26,27], we shall adopt two variants for defining our data set: all the experimental data below $7 \mathrm{TeV}$ (above $5 \mathrm{GeV}$ ) and two independent ensembles by adding either only the TOTEM data at 7, 8 and $13 \mathrm{TeV}$ (ensemble T) or by including also the ATLAS data at 7 and $8 \mathrm{TeV}$ (ensemble $\mathrm{T}+\mathrm{A}$ ). In addition, in order to investigate and stress the importance of the uncertainty regions in the fit results, we shall consider data reductions with two different CL, associated with both one and two standard deviations $(\sigma)$, namely $68.27 \%$ and $95.45 \%$ CL, respectively.
Taking into account the aforementioned critical remarks related to the LHC data, as well as, within the theoretical and experimental uncertainties and both ensembles, our main conclusions from the data reductions are the following: (1) the general analytic model and three particular cases cannot describe, simultaneously, the $\sigma_{\text {tot }}$ and $\rho$ data at $13 \mathrm{TeV}$; (2) one particular Pomeron model, with only seven free parameters and associated with double and triple poles, seems not to be excluded by the bulk of experimental data presently available; (3) for this Pomeron model additional tests on the effect of the subtraction constant and the energy cutoff for data reductions, select the constant as a free fit parameter and cutoff at $5 \mathrm{GeV}$. These results corroborate our previous conclusion [26], now with $1 \sigma$ and $2 \sigma$.

The manuscript is organized as follows. The analytic models are introduced in Sec. II and the fit procedures and results are presented in Sec. III. In Sec. IV, we discuss all the results, and in Sec. V, we present our conclusions and final remarks. In an Appendix, we address some additional tests together with discussions on the results.

\section{ANALYTIC MODELS}

As noted in our Introduction, in the Regge-Gribov theory, simple, double, and triple poles in the complex angular momentum plane are associated with power, logarithmic and logarithmic squared functions of the energy for the total cross section. In this context, for $p p$ and $\bar{p} p$ scattering, we consider a general parametrization for $\sigma_{\text {tot }}(s)$ consisting of two Reggeons (even and odd under crossing) and four (even) Pomeron contributions:

$$
\begin{aligned}
\sigma_{\mathrm{tot}}(s)= & a_{1}\left[\frac{s}{s_{0}}\right]^{-b_{1}}+\tau a_{2}\left[\frac{s}{s_{0}}\right]^{-b_{2}}+A+B\left[\frac{s}{s_{0}}\right]^{\epsilon} \\
& +C \ln \left(\frac{s}{s_{0}}\right)+D \ln ^{2}\left(\frac{s}{s_{0}}\right),
\end{aligned}
$$

where $\tau=-1$ for $p p, \tau=+1$ for $\bar{p} p$, while $a_{1}, b_{1}, a_{2}, b_{2}$, are free fit parameters associated with the secondary Reggeons, $A, B, \epsilon, C, D$ are the free parameters associated with Pomeron components and $s_{0}$ is an energy scale to be discussed in what follows.

The analytic results for $\rho(s)$ have been obtained by means of singly subtracted derivative dispersion relations [28], taking into account an effective subtraction constant $K$ :

$$
\begin{aligned}
\rho(s)= & \frac{1}{\sigma_{\text {tot }}(s)}\left\{\frac{K}{s}-a_{1} \tan \left(\frac{\pi b_{1}}{2}\right)\left[\frac{s}{s_{0}}\right]^{-b_{1}}\right. \\
& +\tau a_{2} \cot \left(\frac{\pi b_{2}}{2}\right)\left[\frac{s}{s_{0}}\right]^{-b_{2}} \\
& \left.+B \tan \left(\frac{\pi \epsilon}{2}\right)\left[\frac{s}{s_{0}}\right]^{\epsilon}+\frac{\pi C}{2}+\pi D \ln \left(\frac{s}{s_{0}}\right)\right\} .
\end{aligned}
$$


As discussed in detail in Appendix C of [7] (and quoted references), $K$ avoids the full high-energy approximation in dispersion relation approaches.

Here, following $[7,15,26,27]$, we consider the energy scale fixed at the physical threshold for scattering states,

$$
s_{0}=4 m_{p}^{2} \sim 3.521 \mathrm{GeV}^{2},
$$

where $m_{p}$ is the proton mass (see Sec. 4.3 in [29] for discussions on this choice).

Equations (4) and (5) bring enclosed analytic structures similar to those appearing in some well known models, as for example, Donnachie and Landshoff (DL) [8], Block and Halzen (BH) [30], COMPETE and PDG parametrizations (COMPETE) [10-12]. We shall consider four particular cases, distinguished by the corresponding Pomeron contributions $\left(\sigma^{P}\right)$, defined and denoted as follows.

(i) Model I: $A=C=D=0 \Rightarrow \sigma_{\mathrm{I}}^{P}=B\left[\frac{s}{s_{0}}\right]^{e}$ (DL-type)

(ii) Model II: $B=C=0, \epsilon=0 \Rightarrow \sigma_{\mathrm{II}}^{P}=A+D \ln ^{2}\left(\frac{s}{s_{0}}\right)$ (COMPETE-type)

(iii) Model III: $A=B=0, \epsilon=0 \Rightarrow \sigma_{\mathrm{III}}^{P}=C \ln \left(\frac{s}{s_{0}}\right)+$ $D \ln ^{2}\left(\frac{s}{s_{0}}\right)($ BH-type $)$

(iv) Model IV: $A=D=0 \Rightarrow \sigma_{\mathrm{IV}}^{P}=B\left[\frac{s}{s_{0}}\right]^{\epsilon}+C \ln \left(\frac{s}{s_{0}}\right)$ (hybrid power-log)

We note that models II and III are analytically similar. The difference concerns the phenomenological interpretation of the singularities as single and double poles. Also, in the $\mathrm{BH}$ analyses, the energy scale is fixed (as we consider here), and in the COMPETE case, it is treated as a free fit parameter.

As far as we know, model IV was never considered in the literature. Its use here is related to tests on the power law (single pole) in the attempt to describe simultaneously the $\sigma_{\text {tot }}$ and the $\rho$ data at $13 \mathrm{TeV}$. We shall return to this point in Sec. IV.B.

In the general model, Eqs. (4) and (5), we have 10 free parameters, $a_{1}, b_{1}, a_{2}, b_{2}, A, B, \epsilon, C, D$ and $K$, which are determined through fits to the experimental data on $\sigma_{\text {tot }}$ and $\rho$ from $p p$ and $\bar{p} p$ elastic scattering in the interval $5 \mathrm{GeV}-13 \mathrm{TeV}$.

\section{FITS AND RESULTS}

\section{A. Ensembles and data reductions}

The data above $5 \mathrm{GeV}$ and below $7 \mathrm{TeV}$ have been collected from the PDG database [12], without any kind of data selection or sieve procedure (we have used all the published data by the experimental collaborations). The data at 7 and $8 \mathrm{TeV}$ by the TOTEM and ATLAS Collaborations can be found in [7], Table 1, together with further information and complete list of references. The TOTEM data at $13 \mathrm{TeV}$ are those in (3) $[13,14]$.

As commented in our introduction, given the tension between the TOTEM and ATLAS measurements on $\sigma_{\text {tot }}$ at $7 \mathrm{TeV}$ and mainly $8 \mathrm{TeV}$, we shall consider two ensembles of $p p$ and $\bar{p} p$ data above $5 \mathrm{GeV}$, both comprising the same dataset in the region below $7 \mathrm{TeV}$. We then construct:

(i) Ensemble TOTEM (T) by adding only the TOTEM data at 7, 8, and $13 \mathrm{TeV}$;

(ii) Ensemble TOTEM + ATLAS $(\mathrm{T}+\mathrm{A})$ by adding to ensemble $\mathrm{T}$ the ATLAS data at 7 and $8 \mathrm{TeV}$.

The fits were performed with the objects of the TMinuit package and using the default MINUIT error analysis [31]. We have carried out global fits using a $\chi^{2}$ fitting procedure, where the value of $\chi_{\min }^{2}$ is distributed as a $\chi^{2}$ distribution with $\nu$ degrees of freedom. The global fits to $\sigma_{\text {tot }}$ and $\rho$ data were performed adopting an interval $\chi^{2}-\chi_{\min }^{2}$ corresponding, in the case of normal errors, to the projection of the $\chi^{2}$ hypersurface containing first $\sim 68 \%$ of probability, and in a second step, $\sim 95 \%$ of probability, namely $1 \sigma$ and $2 \sigma$.

As convergence criteria, we consider only minimization results that imply positive-definite covariance matrices, since theoretically the covariance matrix for a physically motivated function must be positive-definite at the minimum. As tests of goodness-of-fit we shall adopt the chisquare per degree of freedom $\chi^{2} / \nu$ and the integrated probability $P\left(\chi^{2}\right)$ [32].

\section{B. Fit results}

The data reductions with the general model given by Eqs. (4) and (5) did not comply with the above convergence requirements and thus can not be regarded as a possible solution. This may be due to an excessive number of free parameters. On the other hand, in the particular cases given by models I, II, III, and IV, the convergence criteria were reached.

In each case, the values of the free fit parameters with uncertainty of $1 \sigma$, together with the corresponding statistical information, are displayed in Table I in case of ensemble $\mathrm{T}$ and Table II within ensemble $\mathrm{T}+\mathrm{A}$.

Through error propagation from the fit parameters, we determine the uncertainty regions for the theoretical results (curves), within $1 \sigma$ and $2 \sigma$. The results for $\sigma_{\text {tot }}(s)$ and $\rho(s)$ with models I, II, III, and IV (ensembles T and T + A) are compared with the experimental data in Figs. 1-4, respectively. In each figure, the insets highlight the LHC energy region.

\section{DISCUSSION}

On the basis of the fit results in Tables I and II and Figs. 1-4, let us first separate our discussion into two topics related to ensembles (subsection A) and models (subsection B). After that, we shall discuss a selected model in more detail (subsection C).

\section{A. Ensembles $\mathbf{T}$ and $\mathbf{T}+\mathbf{A}$}

Ensemble $\mathrm{T}+\mathrm{A}$ encompasses all the experimental data presently available on forward $p p$ and $\bar{p} p$ scattering at high energies. However, as commented in our introduction and 
TABLE I. Fit results to $\sigma_{\text {tot }}$ and $\rho$ data from ensemble T through models I-IV (Sec. II), by considering one standard deviation, energy cutoff at $5 \mathrm{GeV}$ and $K$ as a free fit parameter.

\begin{tabular}{lcccc}
\hline \hline Model & I & II & III & IV \\
\hline$a_{1}(\mathrm{mb})$ & $41.4 \pm 1.8$ & $32.2 \pm 1.8$ & $58.8 \pm 1.5$ & $51.5 \pm 7.1$ \\
$b_{1}$ & $0.378 \pm 0.028$ & $0.392 \pm 0.049$ & $0.229 \pm 0.017$ & $0.296 \pm 0.037$ \\
$a_{2}(\mathrm{mb})$ & $17.0 \pm 2.0$ & $17.0 \pm 2.1$ & $16.9 \pm 2.0$ & $17.0 \pm 2.1$ \\
$b_{2}$ & $0.545 \pm 0.037$ & $0.545 \pm 0.037$ & $0.543 \pm 0.036$ & $0.544 \pm 0.037$ \\
$A(\mathrm{mb})$ & $\ldots$ & $29.6 \pm 1.2$ & $\ldots$ & $\ldots$ \\
$B(\mathrm{mb})$ & $21.62 \pm 0.73$ & $\ldots$ & $\ldots$ & $9.6 \pm 7.5$ \\
$\epsilon$ & $0.0914 \pm 0.0030$ & $\ldots$ & $\ldots$ & $0.108 \pm 0.019$ \\
$C(\mathrm{mb})$ & $\ldots$ & $\ldots$ & $3.67 \pm 0.34$ & $2.4 \pm 1.6$ \\
$D(\mathrm{mb})$ & $\ldots$ & $0.251 \pm 0.010$ & $0.132 \pm 0.024$ & $\ldots$ \\
$K(\mathrm{mbGeV})$ & $69 \pm 47$ & $55 \pm 50$ & $20 \pm 44$ & $45 \pm 47$ \\
$\nu$ & 248 & 248 & 248 & 247 \\
$\chi^{2} / \nu$ & 1.273 & 1.193 & 1.210 & 1.249 \\
$P\left(\chi^{2}\right)$ & $2.3 \times 10^{-3}$ & $2.0 \times 10^{-2}$ & $1.4 \times 10^{-2}$ & $4.8 \times 10^{-3}$ \\
Figure & 1 & 2 & 3 & 4 \\
\hline \hline
\end{tabular}

discussed in [15,27], the TOTEM and ATLAS data at 7 and $8 \mathrm{TeV}$ present discrepant values. In special, at $8 \mathrm{TeV}$, the ATLAS measurement of $\sigma_{\text {tot }}$ differs from the latest TOTEM result at this energy by 3 standard deviation,

$$
\frac{\sigma_{\mathrm{tot}}^{\mathrm{TOTEM}}-\sigma_{\mathrm{tot}}^{\mathrm{ATLAS}}}{\Delta \sigma_{\mathrm{tot}}^{\mathrm{TOTEM}}}=\frac{103-96.07}{2.3}=3.0
$$

On the one hand, TOTEM published four measurements at $7 \mathrm{TeV}$ and five at $8 \mathrm{TeV}$ (all consistent among them) and ATLAS only one point at each energy. On the other hand, the ATLAS uncertainties in these results are much smaller then the TOTEM uncertainties. For example, at $8 \mathrm{TeV}$, if the ATLAS uncertainty is considered, the aforementioned ratio results 7.5 standard deviation. Besides the TOTEM results for $\sigma_{\text {tot }}$ being larger than the ATLAS values at 7 and $8 \mathrm{TeV}$, the TOTEM data indicate a rise of the total cross section faster than the ATLAS data [15].

Obviously, these facts make any amplitude analyses more difficult and put serious limitations in secure interpretations of the results and unquestionable conclusions that may be reached. It is expected that these discrepancies might be resolved through further re-analyses and/or new data, but it can also happen that these systematic differences may persist. We recall the discrepancies characterizing the experimental information at the highest energy reached in $\bar{p} p$ scattering, namely $1.8 \mathrm{TeV}$. The CDF and E710 results differ by 2.3 standard deviation (respect the E710 uncertainty) and predictions from most phenomenological models lies between these points.

TABLE II. Fit results to $\sigma_{\text {tot }}$ and $\rho$ data from ensemble $\mathrm{T}+\mathrm{A}$ through models I-IV (Sec. II), by considering one standard deviation, energy cutoff at $5 \mathrm{GeV}$ and $K$ as a free fit parameter.

\begin{tabular}{lcccc}
\hline \hline Model & I & II & III & IV \\
\hline$a_{1}(\mathrm{mb})$ & $41.4 \pm 1.8$ & $32.3 \pm 2.0$ & $59.1 \pm 1.5$ & $53.1 \pm 9.6$ \\
$b_{1}(\mathrm{mb})$ & $0.386 \pm 0.028$ & $0.412 \pm 0.045$ & $0.234 \pm 0.016$ & $0.291 \pm 0.044$ \\
$a_{2}(\mathrm{mb})$ & $17.0 \pm 2.1$ & $17.0 \pm 2.0$ & $16.9 \pm 2.0$ & $17.0 \pm 2.1$ \\
$b_{2}(\mathrm{mb})$ & $0.545 \pm 0.037$ & $0.545 \pm 0.036$ & $0.543 \pm 0.036$ & $0.544 \pm 0.038$ \\
$A(\mathrm{mb})$ & $\ldots$ & $30.20 \pm 0.90$ & $\ldots$ & $\ldots$ \\
$B(2.01 \pm 0.64$ & $\ldots$ & $\ldots$ & $8.0 \pm 10$ \\
$\epsilon(\mathrm{mb})$ & $0.0895 \pm 0.0024$ & $\ldots$ & $\ldots$ & $0.110 \pm 0.033$ \\
$D(\mathrm{mb})$ & $\ldots$ & $\ldots$ & $3.81 \pm 0.30$ & $2.8 \pm 2.1$ \\
$K(\mathrm{mbGeV})$ & $73 \pm 48$ & $64 \pm 0.0077$ & $0.119 \pm 0.020$ & $\ldots$ \\
$\nu$ & 250 & 250 & $23 \pm 43$ & $46 \pm 48$ \\
$\chi^{2} / \nu$ & 1.307 & 1.227 & 250 & 249 \\
$P\left(\chi^{2}\right)$ & $7.9 \times 10^{-4}$ & $8.2 \times 10^{-3}$ & $6.9 \times 10^{-3}$ & $2.3 \times 10^{-3}$ \\
Figure & 1 & 2 & 3 & 4 \\
\hline \hline
\end{tabular}


In any case, presently we understand that ensemble $\mathrm{T}+\mathrm{A}$ is the effective representative of the experimental information available, so that an efficient model should be able to access all points within the corresponding uncertainties. More precisely, the predicted uncertainty region must present agreement with the error bars of the experimental points, by reaching all of them, even if in a barely way, but never excluding one or another data, namely TOTEM or ATLAS results.

On the basis of these comments and before discussing the efficiency of each model in the fit results, three characteristics of ensembles $\mathrm{T}$ and $\mathrm{T}+\mathrm{A}$ in our data reductions deserve to be highlighted.

(i) From the figures, for all models the main visual difference in the results within ensembles $\mathrm{T}$ and $\mathrm{T}+\mathrm{A}$ concerns $\sigma_{\mathrm{tot}}$ at the highest energies but not $\rho$ at these energies. Indeed, for example, with model I (Fig. 1) the uncertainty region in the fit result for $\sigma_{\text {tot }}$ at $13 \mathrm{TeV}$ within ensemble $\mathrm{T}$ goes through the lower error bar, the central value and
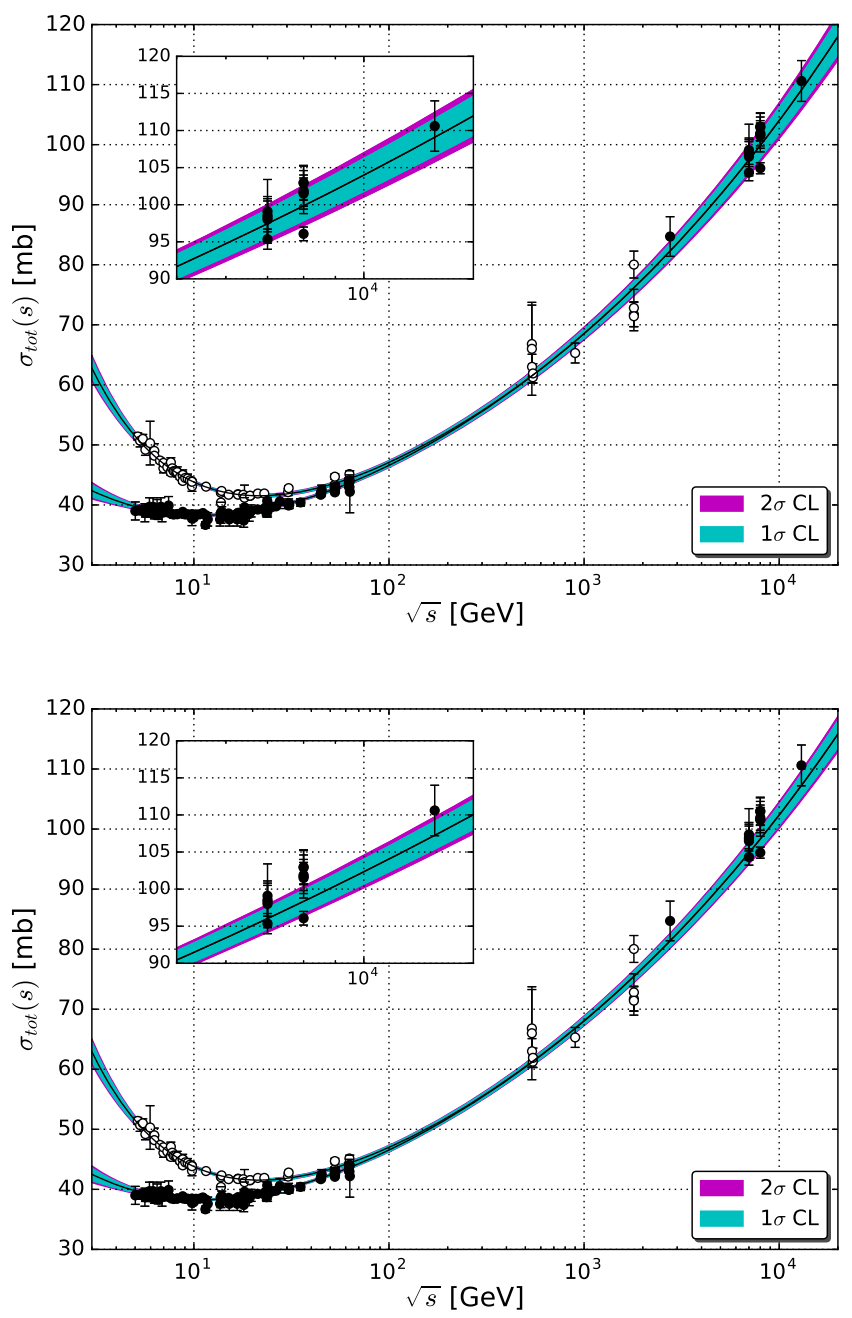

half of the upper error bar, but within ensemble $\mathrm{T}+\mathrm{A}$, goes through only the lower error bar; on the other hand, for $\rho$ at $13 \mathrm{TeV}$ the uncertainty regions within $\mathrm{T}$ and $\mathrm{T}+\mathrm{A}$ are essentially the same, lying far above the experimental data and error bars. Analogous behaviors can be seen in Figs. 2-4. This is a consequence of the large number of experimental data on $\sigma_{\text {tot }}$ at the highest energies (mainly LHC region) as compared with those respect to $\rho$.

(ii) From Tables I and II, in all cases (independently of the ensemble or model), for $\nu \sim 250$, the $\chi^{2} / \nu$ lies in the region $\sim 1.2-1.3$ and the integrated probability $P\left(\chi^{2}\right) \sim 10^{-2}-10^{-3}$. Taking into account the discrepant values between TOTEM and ATLAS data, the fits can be considered as reasonably accurate.

(iii) For models I, II, and III the integrated probability $P\left(\chi^{2}\right)$ is one order of magnitude smaller within ensemble $\mathrm{T}+\mathrm{A}$ than within $\mathrm{T}$ and for model IV nearly $1 / 2$. This is a consequence of the
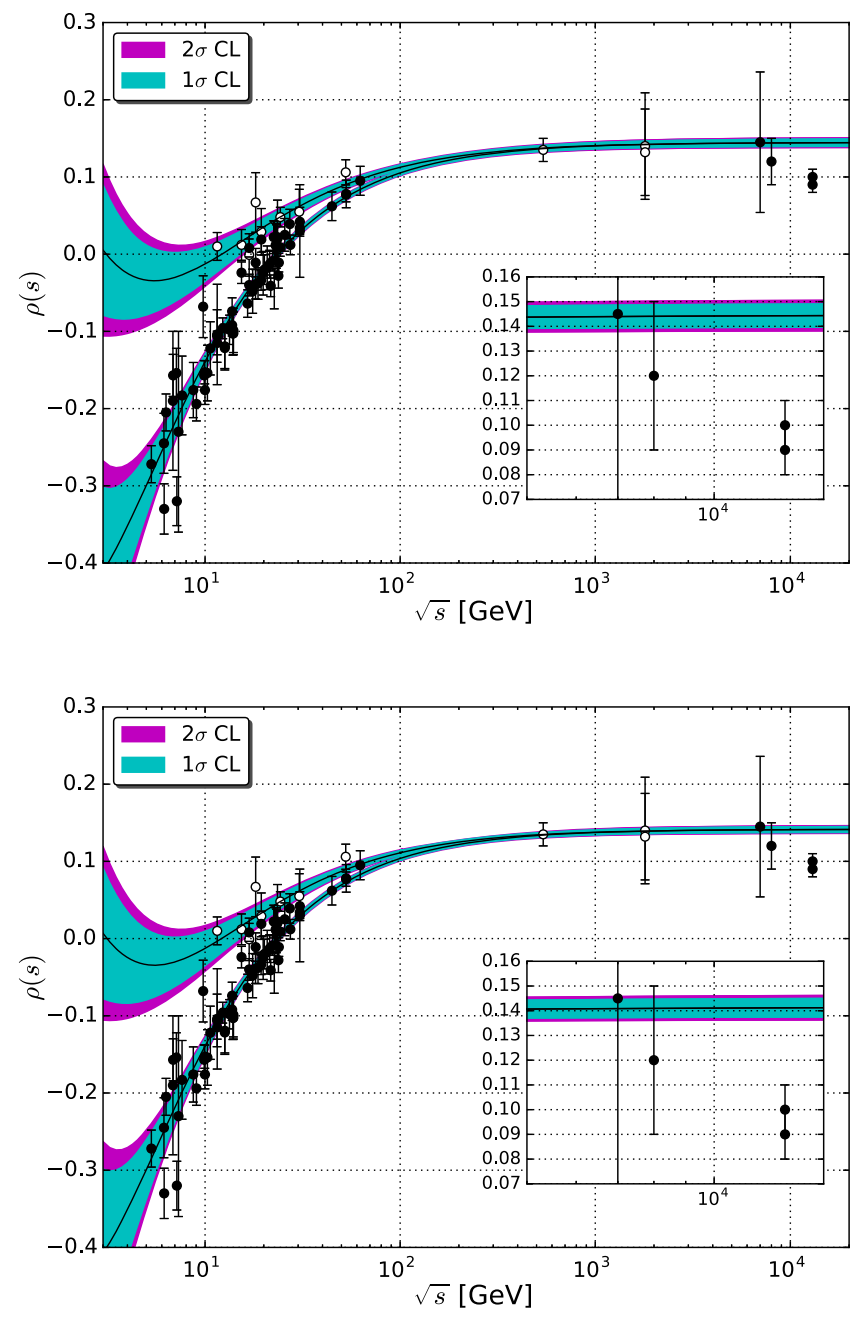

FIG. 1. Fit results with model I to ensembles $\mathrm{T}$ (above) and $\mathrm{T}+\mathrm{A}$ (below). 
aforementioned tension between the TOTEM and ATLAS data at 7 and mainly $8 \mathrm{TeV}$.

\section{B. Models}

First, notice that from the figures and within the uncertainties, all models present quite good descriptions of the experimental data up to $7 \mathrm{TeV}$, as expected. Therefore, let us focus the discussion in the region 8-13 TeV (mainly $13 \mathrm{TeV}$ ) and in the goodness of the fits.

(i) Model I (DL-type)

The fit result in Fig. 1 is in plenty agreement with the $\sigma_{\text {tot }}$ datum at $13 \mathrm{TeV}$ within ensemble $\mathrm{T}$ and the uncertainty region crosses the lower error bar in case of ensemble T + A. However, for $\rho$ the curves do not decrease in the region $10^{3}-10^{4} \mathrm{GeV}$ (see insets) and even with $2 \sigma$ the results at $13 \mathrm{TeV}$ lie far above the upper error bars. Within both ensembles the integrated probability is the smallest among the models $\left(10^{-3}-10^{-4}\right)$. We conclude that this model is not in agreement with the TOTEM data at $13 \mathrm{TeV}$.
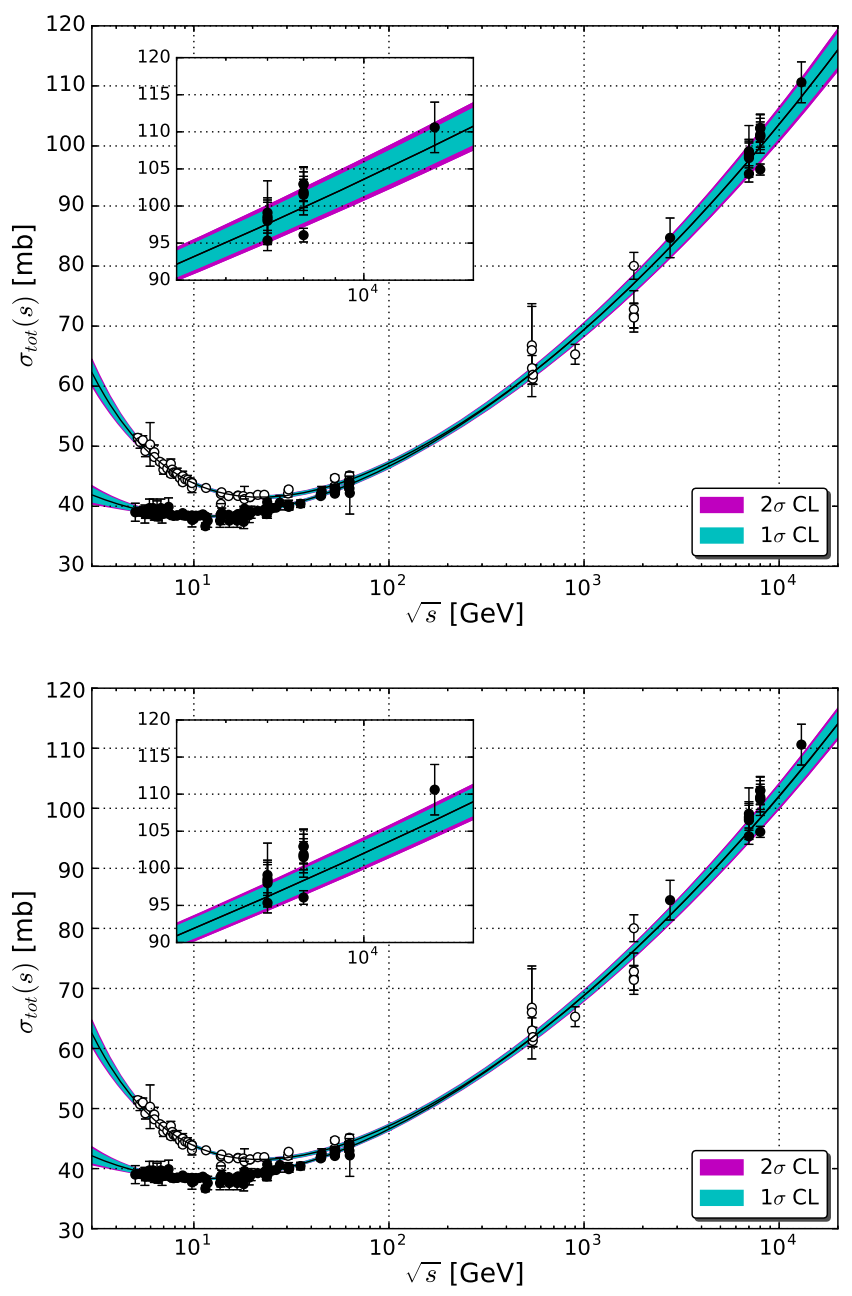

(ii) Model II (COMPETE-type)

From Fig. 2 and ensemble $\mathrm{T}$, the fit result (uncertainty region) for $\sigma_{\text {tot }}$ at $13 \mathrm{TeV}$ crosses the central value and the lower error bar and reaches half this bar within ensemble $\mathrm{T}+\mathrm{A}$. For $\rho$, the curves decrease in the region $10^{3}-10^{4} \mathrm{GeV}$, but as in the previous case, the uncertainty regions lie far above the upper error bars (insets). We conclude this model does not present a satisfactory description of the new data at $13 \mathrm{TeV}$.

(iii) Model III (BH-type)

From the Tables, the integrated probability is one of the highest among the models. From Fig. 3, for $\sigma_{\text {tot }}$ and ensemble $\mathrm{T}+\mathrm{A}$, the uncertainty region with $1 \sigma$ reaches the upper error bar of the ATLAS datum at $8 \mathrm{TeV}$ and the lower bar of the TOTEM datum at $13 \mathrm{TeV}$ (similar with $2 \sigma$ in case of ensemble T). For $\rho$ the curves present the faster decrease among the models in the region $10^{3}-10^{4} \mathrm{GeV}$ (insets) and at $13 \mathrm{TeV}$, with $2 \sigma$,
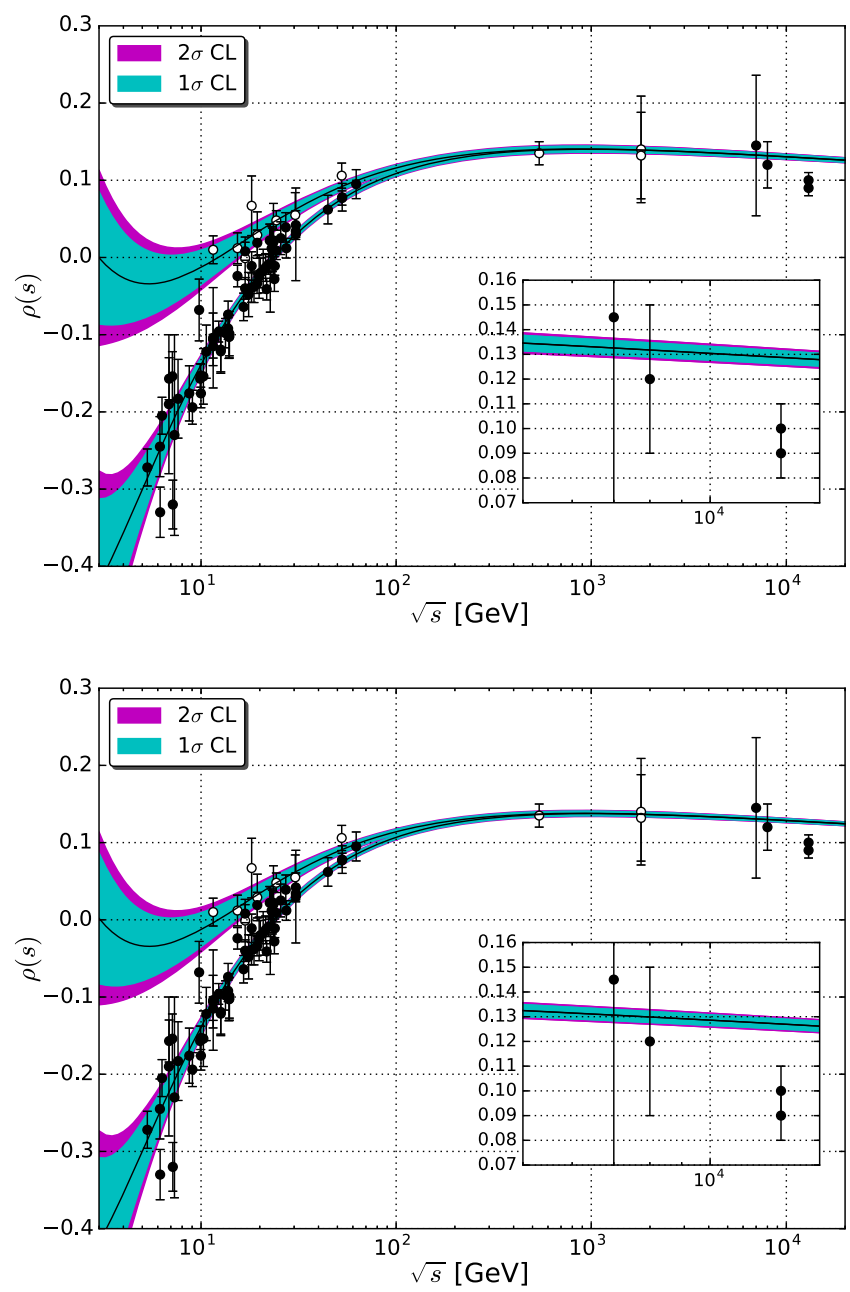

FIG. 2. Fit results with model II to ensembles $\mathrm{T}$ (above) and $\mathrm{T}+\mathrm{A}$ (below). 

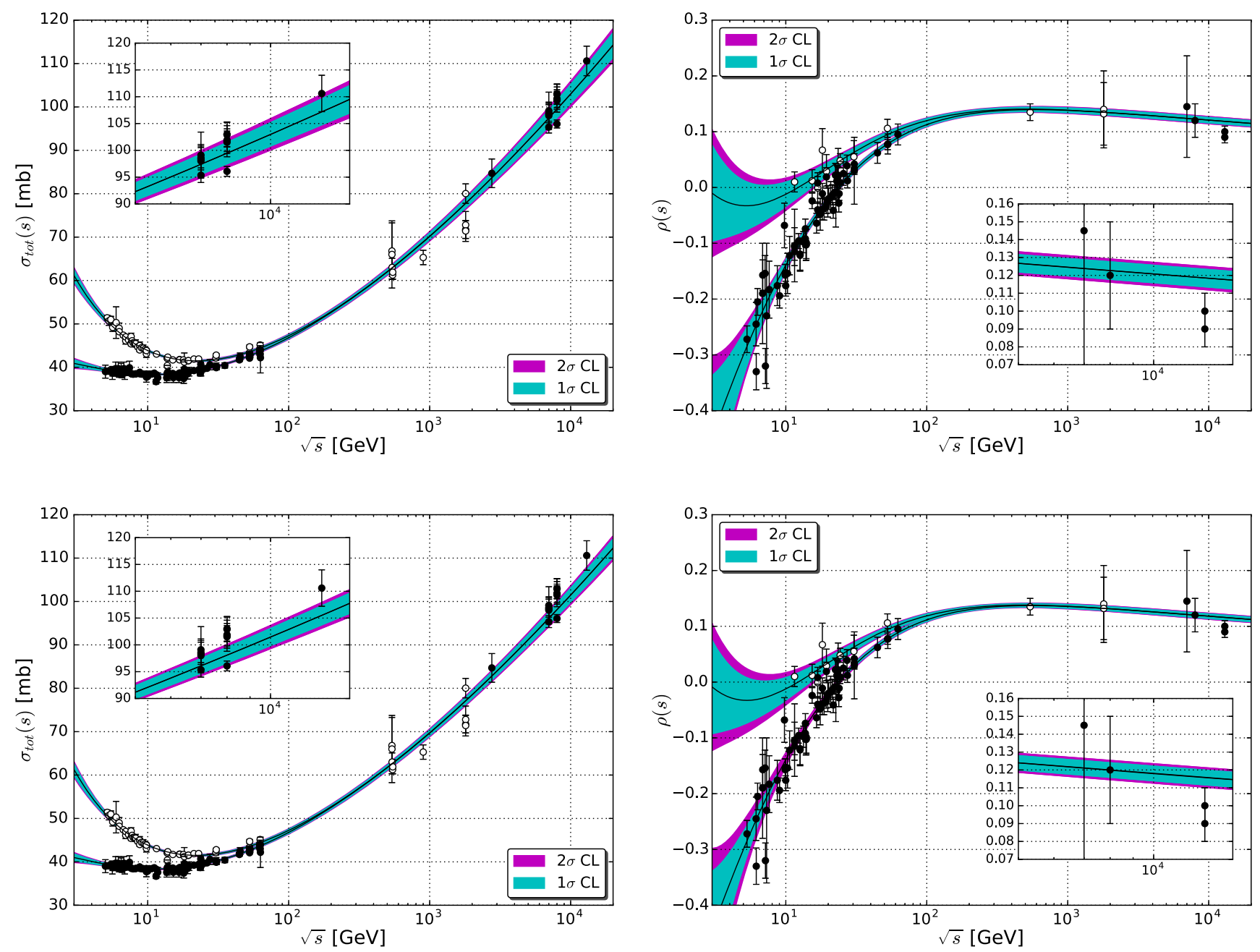

FIG. 3. Fit results with model III to ensembles $\mathrm{T}$ (above) and $\mathrm{T}+\mathrm{A}$ (below).

the uncertainty region reaches the upper extremum of the error bar with ensemble $\mathrm{T}+\mathrm{A}$ (barely reach this point with ensemble $\mathrm{T}$ ). We understand that this model is not excluded by the bulk of experimental data presently available.

(iv) Model IV (Hybrid power-log)

Based on the disagreement of model I with the TOTEM data at $13 \mathrm{TeV}$ and given the efficiency of the power law (simple pole Pomeron) below $13 \mathrm{TeV}$, we have tested hybrid contributions by adding either a double pole or triple pole contributions. In the latter case the fits did not converge and in the former case the fit results are presented in Tables I and II and Fig. 4. In this case, we have one more parameter (as compared with 7 parameters in the other 3 models), resulting in lager uncertainty regions. For $\sigma_{\text {tot }}$ the uncertainty regions with $1 \sigma$ encompass all the experimental data at the LHC energy region. However, from the Tables the integrated probabilities are the smallest among the models and although the results for $\rho$
(Fig. 4) present a small decrease in the region $10^{3}-10^{4} \mathrm{GeV}$, the uncertainty regions lie far above the TOTEM data. We conclude that the model does not present agreement with the TOTEM data at $13 \mathrm{TeV}$.

\section{Conclusions on the Pomeron models and further tests}

Based on the above discussion, we understand that models I, II, and IV are not able to describe simultaneously the TOTEM data on $\sigma_{\text {tot }}$ and $\rho$ at $13 \mathrm{TeV}$. On the other hand, taking into account the bulk of experimental data presently available (ensemble $\mathrm{T}+\mathrm{A}$ ) and the uncertainties in both theoretical and experimental results, model III seems not to be excluded.

Looking for possible improvements in the efficiency of model III, we have also developed further tests with some variants. Here, in all fits we have considered the energy cutoff at $\sqrt{s}_{\min }=5 \mathrm{GeV}$ and the subtraction constant as a free fit parameter. In order to investigate the effect of the 

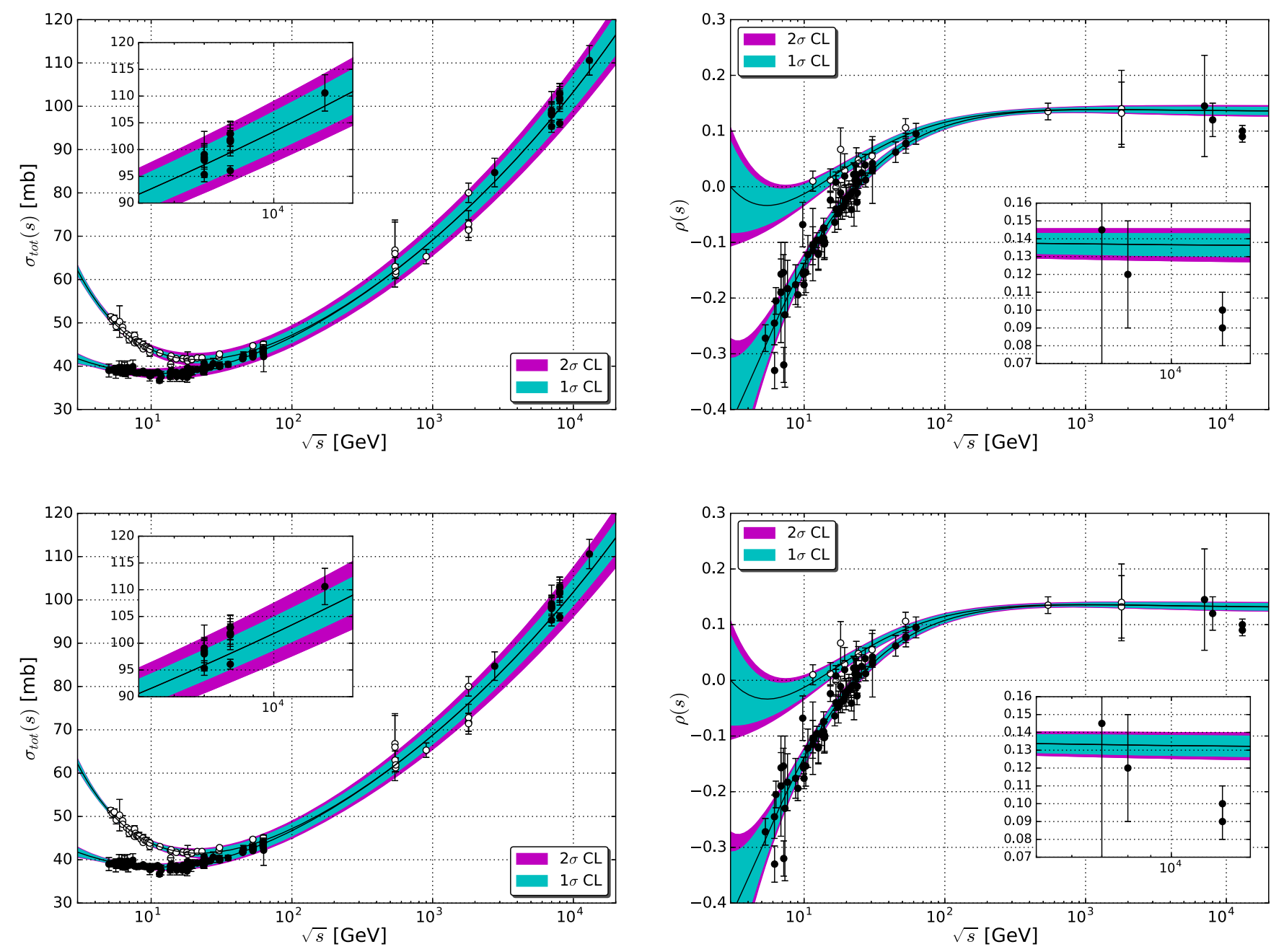

FIG. 4. Fit results with model IV to ensembles $\mathrm{T}$ (above) and $\mathrm{T}+\mathrm{A}$ (below).

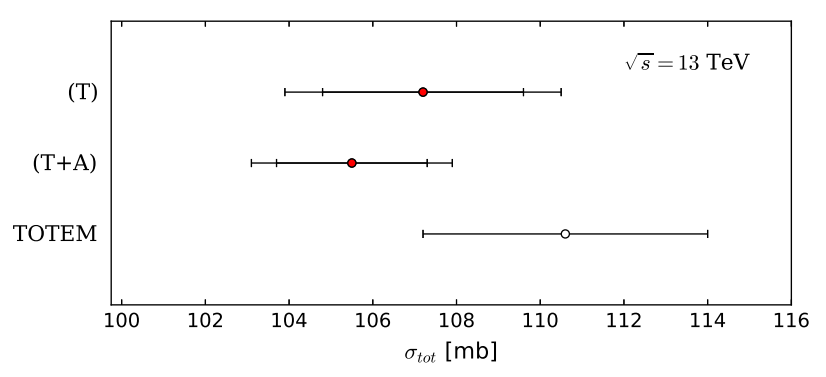

energy cutoff and the role of the subtraction constant we have also carried out fits without this parameter, namely by fixing $K=0$ and rising the energy cutoff to 7.5 and $10 \mathrm{GeV}$. The results are presented in the Appendix, together with a short discussion. Taking into account the energy region analyzed, $5 \mathrm{GeV}-13 \mathrm{TeV}$ and the $p p$ and $\bar{p} p$ scattering, we did not find remarkable or considerable improvements. Indeed, with the cutoff at $5 \mathrm{GeV}$, the results with and without the subtraction constant are similar, with integrated probability slightly greater in case of $K$ free and the uncertainty region reaching the extreme of the upper error bar of $\rho$ at $13 \mathrm{TeV}$ (ensemble $\mathrm{T}+\mathrm{A}$ ).

Therefore, we select as our best result those obtained here with model III, cutoff at $5 \mathrm{GeV}$ and the subtraction constant as a free fit parameter (Fig. 3 and Tables I and II). For this case, we present in Fig. 5 a detail of the predictions for $\sigma_{\text {tot }}$ and $\rho$ at $13 \mathrm{TeV}$ and the experimental data; the numerical values are given in Table III, together with the corresponding predictions at $14 \mathrm{TeV}$ and uncertainties associated with $1 \sigma$ and also $2 \sigma$.

Predictions of model III for $\sigma_{\text {tot }}$ and $\rho$ at $13 \mathrm{TeV}$ with 1 an standard deviations from fits to ensemble Tand $\mathrm{T}+\mathrm{A}$ (filled circles) together with the TOTEM measurements (3) (empty circles). 
TABLE III. Predictions of model III for $\sigma_{\text {tot }}$ and $\rho$ at $13 \mathrm{TeV}$ and $14 \mathrm{TeV}$ for $p p$ and $\bar{p} p$ scattering: central values and uncertainties with $1 \sigma$ and $2 \sigma$ (Tables I and II).

\begin{tabular}{|c|c|c|c|c|c|c|c|}
\hline \multirow[b]{2}{*}{$\sqrt{s}(\mathrm{TeV})$} & \multirow[b]{2}{*}{ Ensemble } & \multicolumn{3}{|c|}{$\sigma_{\text {tot }}(\mathrm{mb})$} & \multicolumn{3}{|c|}{$\rho$} \\
\hline & & Central & $1 \sigma$ & $2 \sigma$ & Central & $1 \sigma$ & $2 \sigma$ \\
\hline 13 & $\begin{array}{c}\mathrm{T} \\
\mathrm{T}+\mathrm{A}\end{array}$ & $\begin{array}{l}107.2 \\
105.5\end{array}$ & $\begin{array}{l} \pm 2.4 \\
\pm 1.8\end{array}$ & $\begin{array}{l} \pm 3.3 \\
\pm 2.4\end{array}$ & $\begin{array}{l}0.1185 \\
0.1158\end{array}$ & $\begin{array}{l} \pm 0.0049 \\
\pm 0.0042\end{array}$ & $\begin{array}{l} \pm 0.0065 \\
\pm 0.0055\end{array}$ \\
\hline 14 & $\begin{array}{c}\mathrm{T} \\
\mathrm{T}+\mathrm{A}\end{array}$ & $\begin{array}{l}108.4 \\
106.7\end{array}$ & $\begin{array}{l} \pm 2.5 \\
\pm 1.8\end{array}$ & $\begin{array}{l} \pm 3.3 \\
\pm 2.5\end{array}$ & $\begin{array}{l}0.1179 \\
0.1152\end{array}$ & $\begin{array}{l} \pm 0.0049 \\
\pm 0.0042\end{array}$ & $\begin{array}{l} \pm 0.0065 \\
\pm 0.0055\end{array}$ \\
\hline
\end{tabular}

\section{CONCLUSIONS AND FINAL REMARKS}

We have presented a forward amplitude analysis on the experimental data presently available from $p p$ and $\bar{p} p$ scattering in the energy region $5 \mathrm{GeV}-13 \mathrm{TeV}$. The analysis consists of tests with different analytic parametrizations for $\sigma_{\text {tot }}(s)$ and $\rho(s)$, all of them characterized by Pomeron leading contributions (even-under-crossing). The data reductions show that most models present no simultaneous agreement with the recent $\sigma_{\text {tot }}$ and $\rho$ measurements at $13 \mathrm{TeV}$ by the TOTEM Collaboration. Different models and variants have been tested and among them, model III (two simple poles Reggeons, one double pole and one triple pole Pomerons), with only seven free fit parameters, led to the best results.

Two aspects have been stressed along the paper. The first concerns the TOTEM results at $13 \mathrm{TeV}$, indicating an expected rise of the total cross section but an unexpected decrease in the value of the $\rho$ parameter. The extrapolation from the recent analysis with data up to $8 \mathrm{TeV}$, discussed in our introduction, shows clearly the plenty agreement with the $\sigma_{\text {tot }}$ result and the overestimation of the $\rho$ data [15]. Note also that the value here obtained for the Pomeron intercept with model I and ensemble T, $\epsilon=0.0914 \pm$ 0.0039 (Table I) is consistent with results of fits up to $8 \mathrm{TeV}$, for example those obtained in [29]: $\epsilon=$ $0.0926 \pm 0.0016$. However, the model I result for $\rho$ at $13 \mathrm{TeV}$ is in complete disagreement with the TOTEM data (Fig. 1).

The second aspect concerns the tension between the TOTEM and ATLAS data at $7 \mathrm{TeV}$ and mainly at $8 \mathrm{TeV}$, discussed in certain detail in the previous sections. That led us to consider separately the two ensembles denoted $\mathrm{T}$ (excluding the ATLAS data) and $\mathrm{T}+\mathrm{A}$ (including the ATLAS data). We have shown that these discrepancies play an important role in the interpretations of the fit results.

Another aspect deserves attention when interpreting the data reductions. As discussed in Appendix A of [7], the TOTEM uncertainties are essentially systematic (uniform distribution) and not statistical (Gaussian distribution). Therefore, a model result crossing the central value of an experimental result may have a limited significance on statistical grounds.
The unexpected decrease in the $\rho$ value has been well described in the recent analyses by Martynov and Nicolescu. The first paper treated only the TOTEM data [20] and in the second one the ATLAS data have been included [21]. The $\chi^{2} / \nu$ are similar in both cases, namely 1.075 without ATLAS and 1.100 including ATLAS, corresponding to an increase of $2.3 \%$. For $\rho$ at $13 \mathrm{TeV}$, in both cases the curves seems to cross the central value of the experimental points (one symbol in Figs. 2 and 3 of [21]). However, for $\sigma_{\text {tot }}$ with ATLAS excluded the curve crosses the lower error bar at $13 \mathrm{TeV}$, but lies above the error bars of the ATLAS data at $7 \mathrm{TeV}$ and mainly $8 \mathrm{TeV}$. With ATLAS included, the curve crosses the ATLAS data, but lies below the lower error bar of the TOTEM data at $8 \mathrm{TeV}$ and mainly $13 \mathrm{TeV}$ (Fig. 3 of [21]). Summarizing, the curve does not reach the upper error bars of the ATLAS data on $\sigma_{\text {tot }}$ at 7 and $8 \mathrm{TeV}$ in the former case and does not reach the lower error bar of the TOTEM datum at $13 \mathrm{TeV}$ in the latter case.

In what concerns our results with model III, the $\chi^{2} / \nu$ are also similar in both cases: $1.210(\mathrm{~T})$ and $1.234(\mathrm{~T}+\mathrm{A})$, corresponding to an increase slightly small, $2.0 \%$. The uncertainty regions of the fit results do not cross the central values of the $\rho$ data at $13 \mathrm{TeV}$, but barely reach the upper error bar. However, the same is true for the ATLAS datum on $\sigma_{\text {tot }}$ at $8 \mathrm{TeV}$. Therefore, we conclude that the agreement between the phenomenological model and the experimental points is reasonably compatible within the uncertainties. In other words, in case of fits to ensembles $\mathrm{T}$ or $\mathrm{T}+\mathrm{A}$ (all the experimental data presently available) and within the uncertainties, the Pomeron model III, with seven free fit parameters, seems not to be excluded by the experimental data presently available on forward $p p$ and $\bar{p} p$ elastic scattering.

In the theoretical context, the Odderon is a well-founded concept in perturbative QCD [18]. Despite the consistent phenomenological description of the unexpected decrease of the $\rho$ parameter at $13 \mathrm{TeV}$, the Odderon model predicts a crossing in the $p p$ and $\bar{p} p$ total cross sections at high energies. Although in agreement with high-energy theorems [33], it seems still lacking a pure (model independent) nonperturbative QCD explanation (from first principles) for an asymptotic rise of the total cross section faster for hadron-hadron than for antihadron-hadron collisions. 
Finally, we understand that further re-analysis and new experimental data at $13 \mathrm{TeV}$ and $14 \mathrm{TeV}$, by the TOTEM and ATLAS collaborations, shall be crucial for confronting, in a conclusive way, the possible dominance of Odderon or Pomeron in forward elastic hadron scattering at high energies.

\section{ACKNOWLEDGMENTS}

This research was partially supported by the Conselho Nacional de Desenvolvimento Científico e Tecnológico (CNPq) Grant No. 141496/2015-0. E. G. S. L. acknowledges the financial support from the Rede Nacional de Altas Energias (RENAFAE).

\section{APPENDIX: FURTHER TESTS WITH MODEL III}

In Sec. III, the fits through model III to ensembles T and $\mathrm{T}+\mathrm{A}$ were carried out with energy cutoff at $5 \mathrm{GeV}$ and the subtraction constant as a free fit parameter (Tables I and II and Fig. 3). In what follows, we consider two variants related to the energy cutoff and the subtraction constant. Firstly, still with the subtraction constant as a free fit parameter, we develop fits with energy cutoff at 7.5 and $10 \mathrm{GeV}$. The results are displayed in Table IV and Figs. 6 and 7. In a second step the subtraction constant is fixed at zero and the fits are developed with energy cutoff at 5, 7.5, and $10 \mathrm{GeV}$. The results are shown in Table V and Figs. 8-10. As before, in all the cases we employ ensembles $\mathrm{T}$ and $\mathrm{T}+\mathrm{A}$ and $\mathrm{CL}$ with one and two standard deviations.

For $\mathrm{K}$ as a free fit parameter, comparison of Table I (cutoff at $5 \mathrm{GeV}$ ) with Table IV (cutoffs at 7.5 and $10 \mathrm{GeV}$ ), shows that for both ensembles, rising the cutoff results in a slightly increase in $P\left(\chi^{2}\right)$ and from Figs. 3, 6, and 7, the uncertainty regions become larger, mainly at lower energies. The same effect is observed by fixing $K=0$ (Table $\mathrm{V}$ and Figs. 8-10). The rise of the cutoff does not led to an improvement in the fit results, within the uncertainty region, at $13 \mathrm{TeV}$.
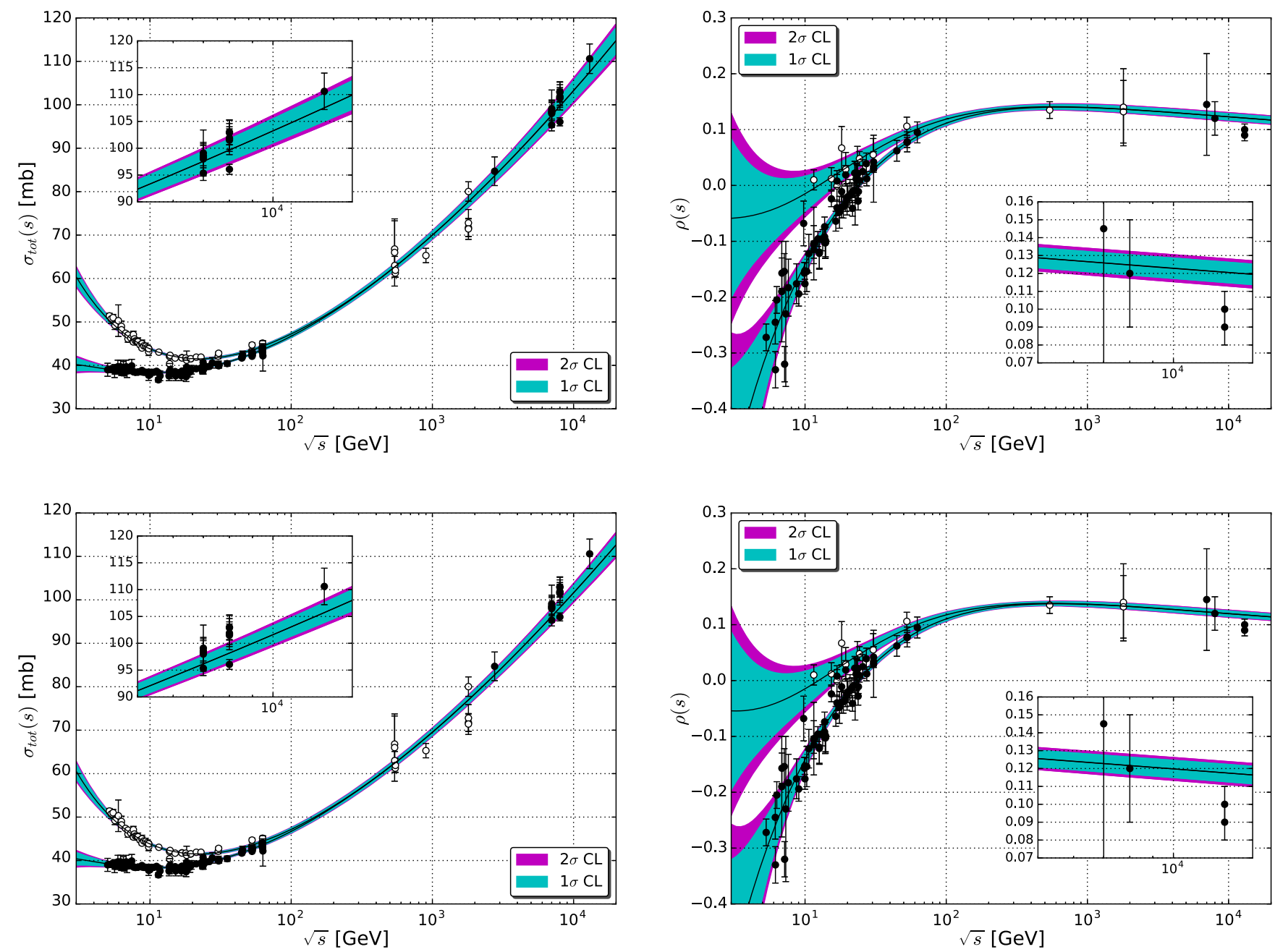

FIG. 6. Fit results with model III to ensembles T (above) and T $+\mathrm{A}$ (below) by considering the energy cutoff at $\sqrt{s}=7.5 \mathrm{GeV}$ and $K$ as a free fit parameter. 

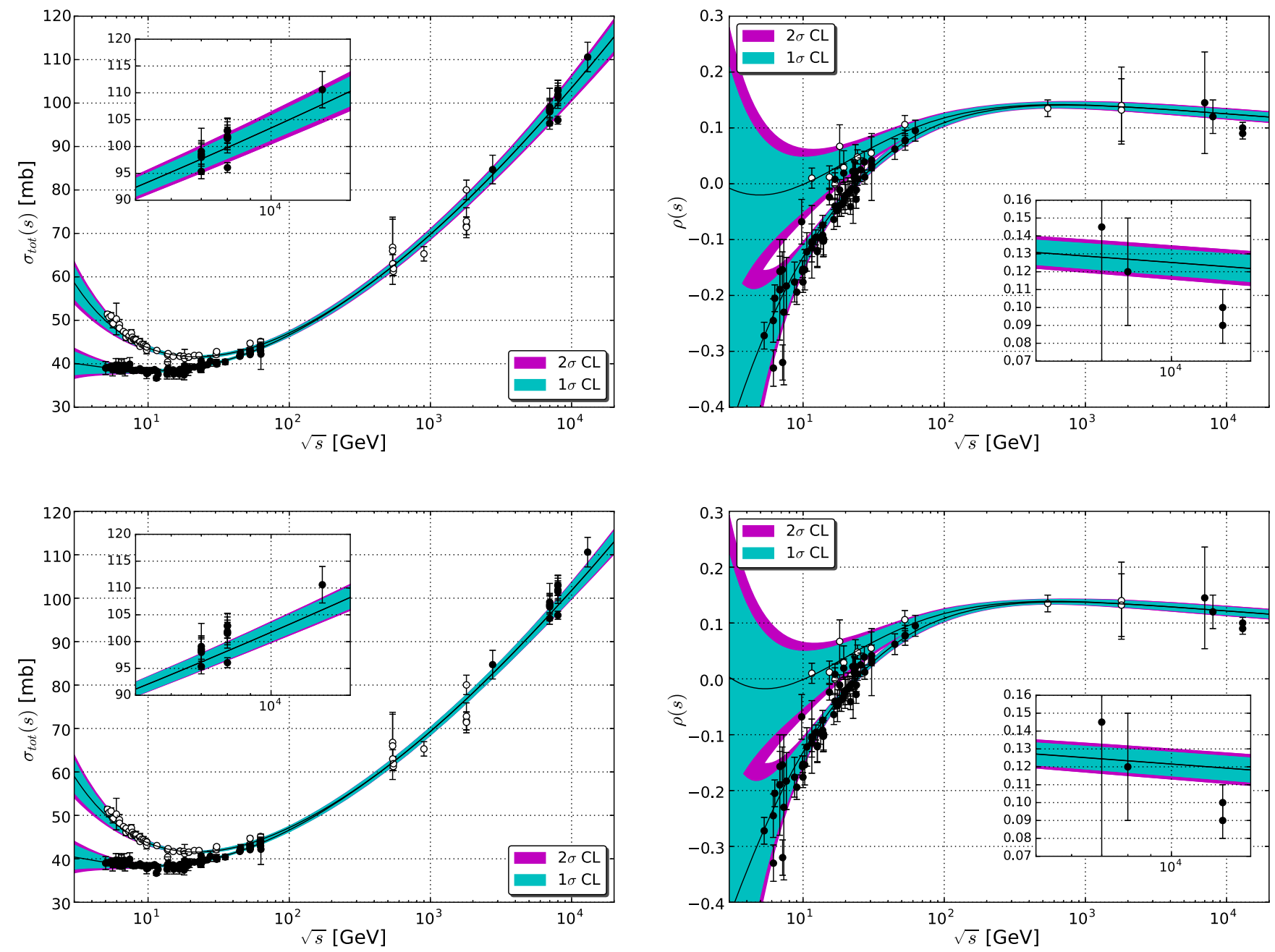

FIG. 7. Fit results with model III to ensembles $\mathrm{T}$ (above) and $\mathrm{T}+\mathrm{A}$ (below) by considering the energy cutoff at $\sqrt{s}=10 \mathrm{GeV}$ and $K$ as a free fit parameter.

TABLE IV. Fit results with model III to ensembles $\mathrm{T}$ and $\mathrm{T}+\mathrm{A}$ by considering one-standard deviation, energy cutoffs at 7.5 and $10 \mathrm{GeV}$ and $K$ as a free fit parameter.

\begin{tabular}{lccccc}
\hline \hline Ensemble & \multicolumn{2}{c}{$\mathrm{T}$} & & \multicolumn{2}{c}{$\mathrm{T}+\mathrm{A}$} \\
\cline { 2 - 3 } \cline { 6 - 6 }$\sqrt{s}_{\min }(\mathrm{GeV})$ & 7.5 & 10 & & 7.5 & 10 \\
\hline$a_{1}(\mathrm{mb})$ & $57.5 \pm 2.1$ & $55.8 \pm 4.0$ & & $57.9 \pm 2.1$ & $56.5 \pm 4.1$ \\
$b_{1}$ & $0.217 \pm 0.023$ & $0.202 \pm 0.037$ & & $0.224 \pm 0.021$ & $0.212 \pm 0.037$ \\
$a_{2}(\mathrm{mb})$ & $16.8 \pm 2.7$ & $15.1 \pm 4.6$ & & $16.8 \pm 2.7$ & $15.1 \pm 4.8$ \\
$b_{2}$ & $0.542 \pm 0.046$ & $0.520 \pm 0.070$ & & $0.542 \pm 0.046$ & $0.520 \pm 0.072$ \\
$C(\mathrm{mb})$ & $3.48 \pm 0.44$ & $3.25 \pm 0.66$ & & $3.66 \pm 0.38$ & $3.48 \pm 0.59$ \\
$D(\mathrm{mb})$ & $0.143 \pm 0.030$ & $0.156 \pm 0.040$ & & $0.128 \pm 0.024$ & $0.138 \pm 0.035$ \\
$\left.K(\mathrm{mbGeV})^{2}\right)$ & $-15 \pm 74$ & $4.17 \pm 116$ & & $-9.5 \pm 73$ & $14.3 \pm 117$ \\
$\nu$ & 205 & 164 & & 207 & 166 \\
$\chi^{2} / \nu$ & 1.217 & 1.213 & & 1.253 & 1.263 \\
$P\left(\chi^{2}\right)$ & $1.8 \times 10^{-2}$ & $3.3 \times 10^{-2}$ & & $7.8 \times 10^{-3}$ & $1.2 \times 10^{-2}$ \\
Figure & 6 & 7 & 6 & 7 \\
\hline \hline
\end{tabular}


TABLE V. Fit results with model III to ensembles $\mathrm{T}$ and $\mathrm{T}+\mathrm{A}$ by considering one-standard deviation, energy cutoffs at $5,7.5$ and $10 \mathrm{GeV}$ and the subtraction constant $K=0$ (fixed).

\begin{tabular}{|c|c|c|c|c|c|c|}
\hline \multirow{2}{*}{$\begin{array}{l}\text { Ensemble } \\
{\sqrt{s_{\min }}}(\mathrm{GeV})\end{array}$} & \multicolumn{3}{|c|}{$\mathrm{T}$} & \multicolumn{3}{|c|}{$\mathrm{T}+\mathrm{A}$} \\
\hline & 5 & 7.5 & 10 & 5 & 7.5 & 10 \\
\hline$a_{1}(\mathrm{mb})$ & $58.6 \pm 1.3$ & $57.7 \pm 1.8$ & $55.8 \pm 3.1$ & $58.8 \pm 1.3$ & $58.0 \pm 1.8$ & $56.2 \pm 3.1$ \\
\hline$b_{1}$ & $0.226 \pm 0.015$ & $0.219 \pm 0.019$ & $0.202 \pm 0.029$ & $0.231 \pm 0.014$ & $0.225 \pm 0.018$ & $0.209 \pm 0.028$ \\
\hline$a_{2}(\mathrm{mb})$ & $17.0 \pm 1.8$ & $16.6 \pm 2.3$ & $15.2 \pm 4.2$ & $17.1 \pm 1.8$ & $16.6 \pm 2.3$ & $15.3 \pm 4.3$ \\
\hline$b_{2}$ & $0.547 \pm 0.032$ & $0.538 \pm 0.038$ & $0.521 \pm 0.064$ & $0.548 \pm 0.032$ & $0.539 \pm 0.038$ & $0.522 \pm 0.063$ \\
\hline$C(\mathrm{mb})$ & $3.62 \pm 0.30$ & $3.51 \pm 0.38$ & $3.24 \pm 0.53$ & $3.76 \pm 0.26$ & $3.67 \pm 0.33$ & $3.44 \pm 0.47$ \\
\hline$D(\mathrm{mb})$ & $0.135 \pm 0.022$ & $0.141 \pm 0.026$ & $0.157 \pm 0.033$ & $0.122 \pm 0.018$ & $0.127 \pm 0.021$ & $0.140 \pm 0.029$ \\
\hline$\nu$ & 249 & 206 & 165 & 251 & 208 & 167 \\
\hline$\chi^{2} / \nu$ & 1.210 & 1.213 & 1.206 & 1.238 & 1.248 & 1.256 \\
\hline$P\left(\chi^{2}\right)$ & $1.3 \times 10^{-2}$ & $2.0 \times 10^{-2}$ & $3.7 \times 10^{-2}$ & $6.1 \times 10^{-3}$ & $8.8 \times 10^{-3}$ & $1.4 \times 10^{-2}$ \\
\hline Figure & 8 & 9 & 10 & 8 & 9 & 10 \\
\hline
\end{tabular}
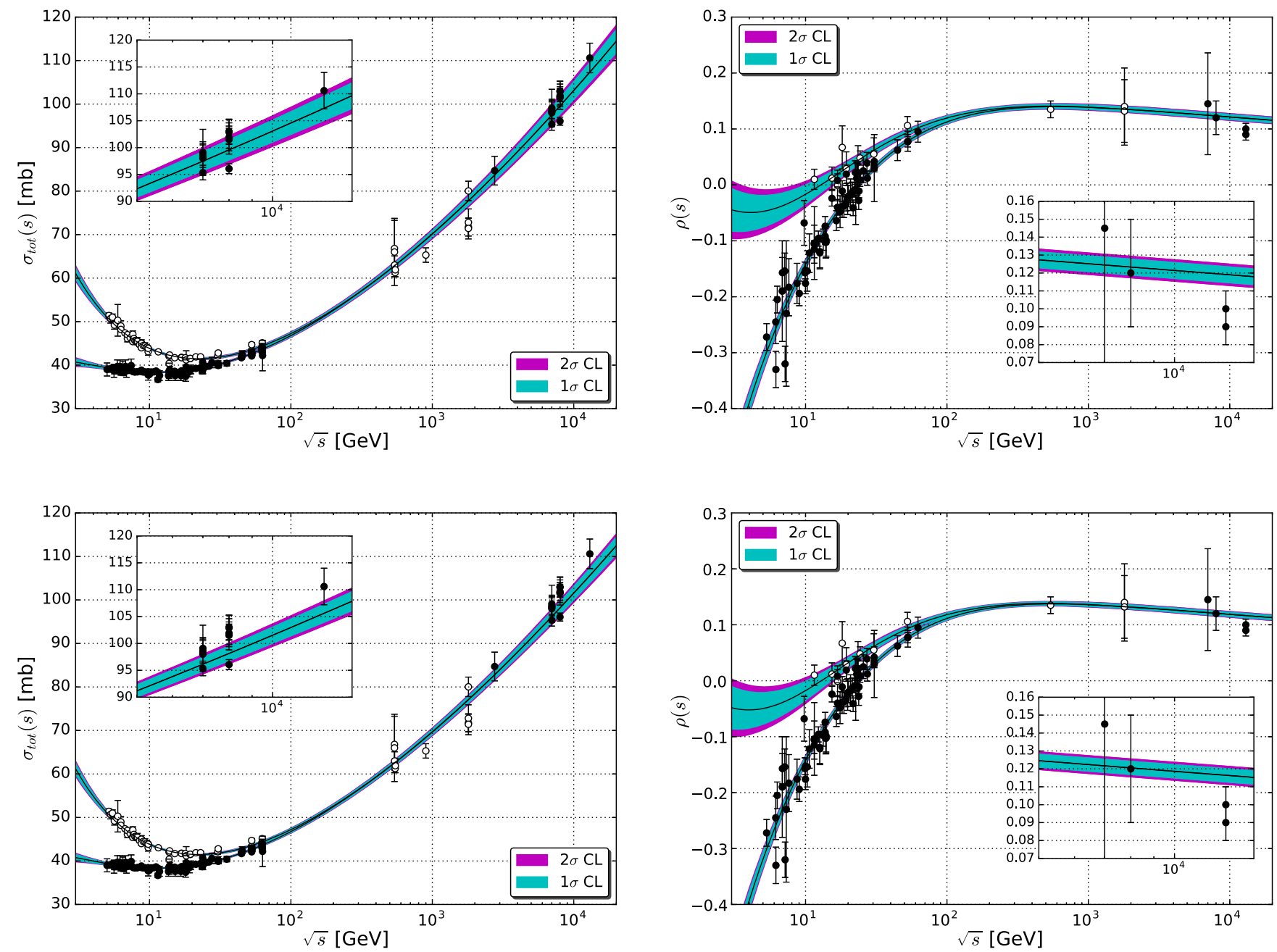

FIG. 8. Fit results with model III to ensembles $\mathrm{T}$ (above) and $\mathrm{T}+\mathrm{A}$ (below) by considering the energy cutoff at $\sqrt{s}=5 \mathrm{GeV}$ and $K=0$ (fixed). 
For cutoff at $5 \mathrm{GeV}$, the results with $K$ free (Table I, Fig. 3) and $K=0$ fixed (Table V, Fig. 8) show the following features:

(i) within both ensembles, the integrated probability is slightly larger for $K$ free;

(ii) for $\rho$ at $13 \mathrm{TeV}$ and ensemble $\mathrm{T}$, the distance between the minimum of the uncertainty region and the extreme of the upper error bar is smaller with $K$ free than with $K=0$;
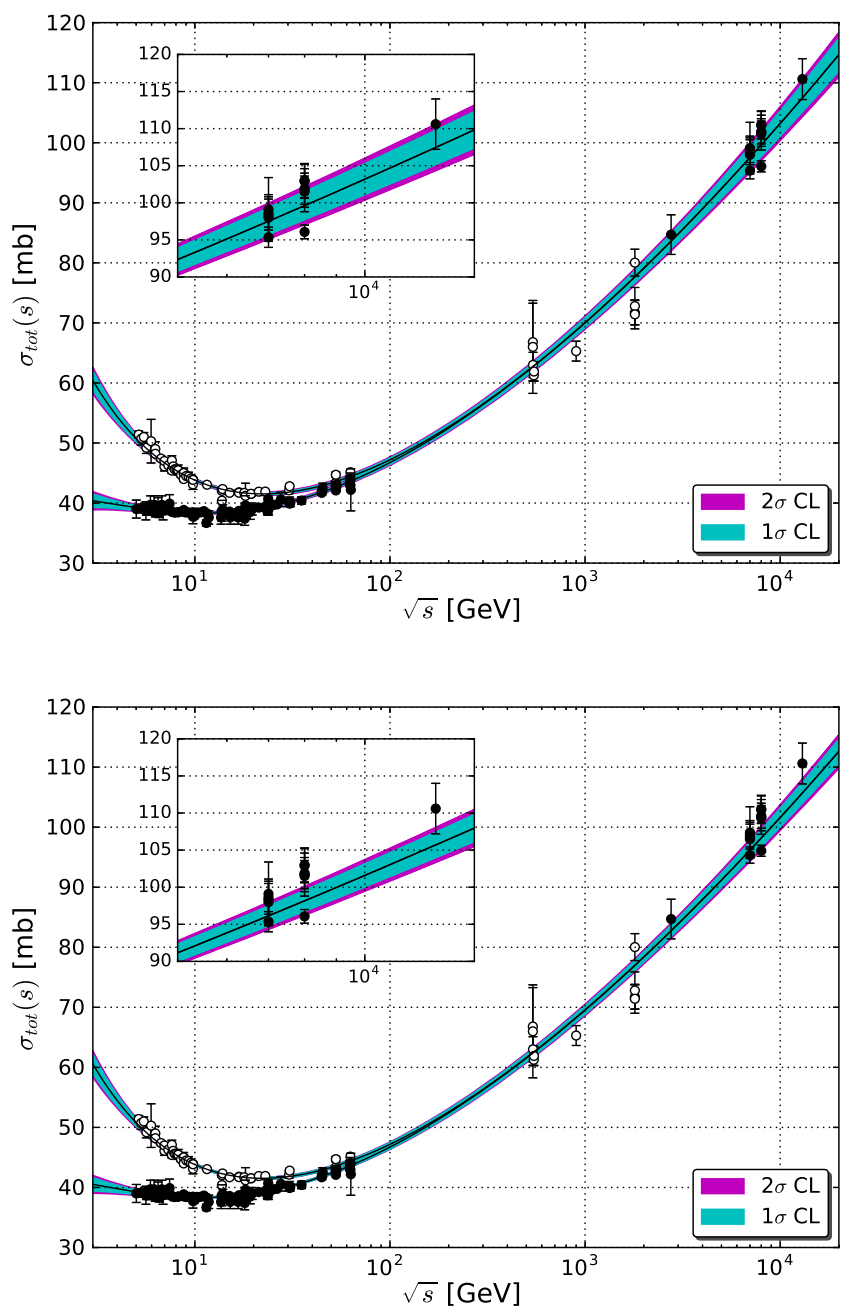

(iii) for $\rho$ at $13 \mathrm{TeV}$, ensemble $\mathrm{T}+\mathrm{A}$ and $K=0$, the uncertainty region lies slightly above the extreme of the upper error bar (Fig. 8) and for $K$ free the uncertainty region reaches this point (Fig. 3).

In conclusion, the rising of the cutoff does not lead to improvements in the fit results, neither fixing $K=0$. The results with $K$ free and cutoff at $5 \mathrm{GeV}$ present best agreement with the TOTEM data at $13 \mathrm{TeV}$.
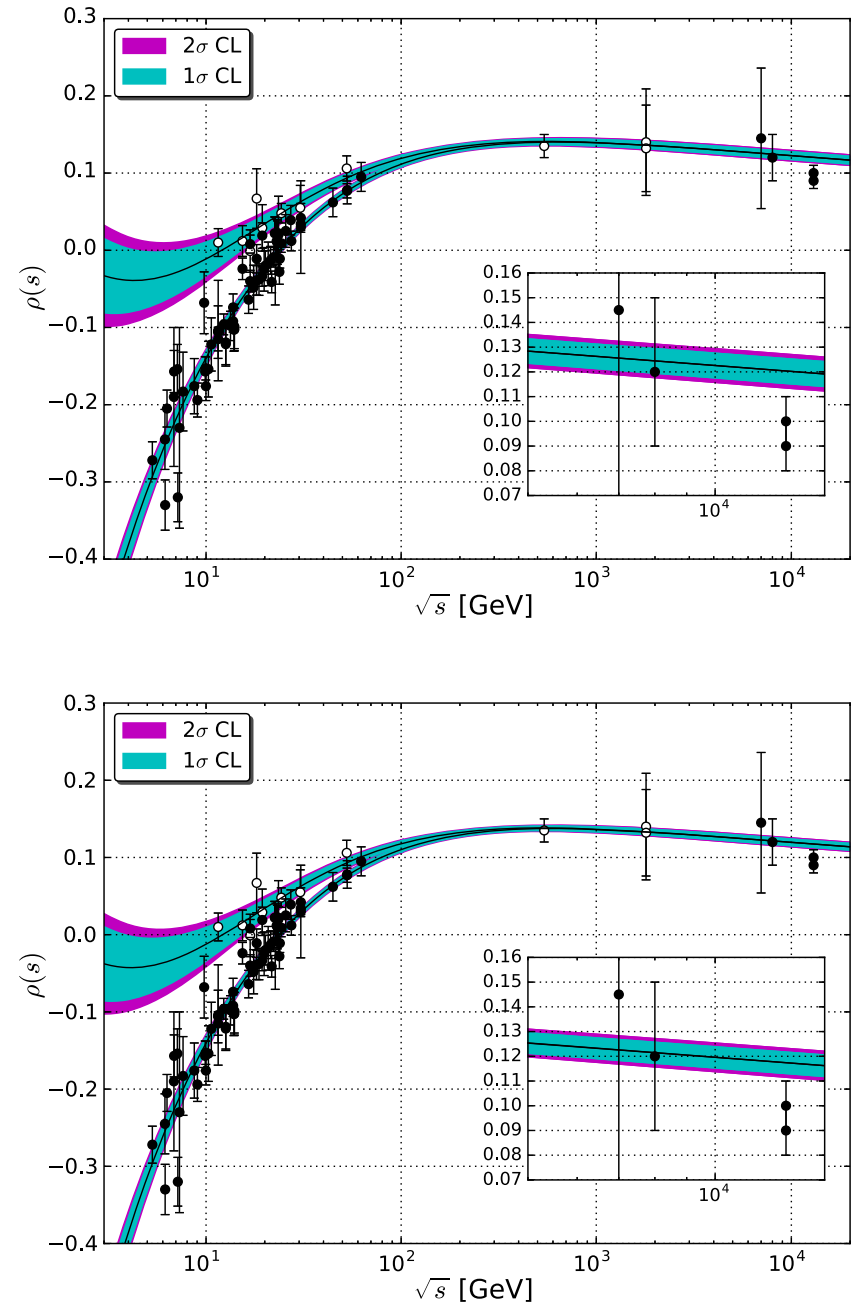

FIG. 9. Fit results with model III to ensembles $\mathrm{T}$ (above) and $\mathrm{T}+\mathrm{A}$ (below) by considering the energy cutoff at $\sqrt{s}=7.5 \mathrm{GeV}$ and $K=0$ (fixed). 

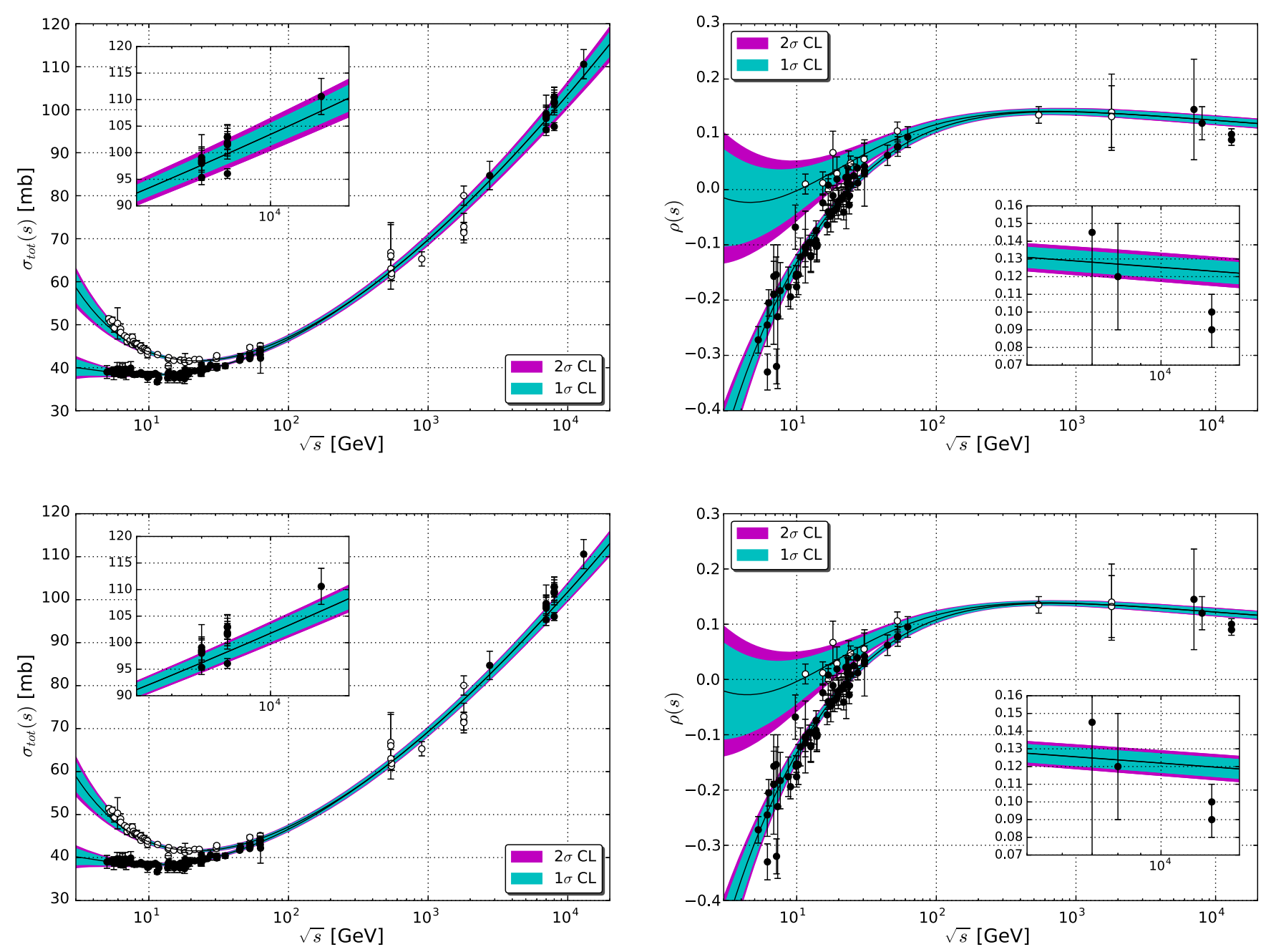

FIG. 10. Fit results with model III to ensembles $\mathrm{T}$ (above) and $\mathrm{T}+\mathrm{A}$ (below) by considering the energy cutoff at $\sqrt{s}=10 \mathrm{GeV}$ and $K=0$ (fixed).

[1] V. Barone and E. Predazzi, High-Energy Particle Diffraction (Springer, New York, 2002).

[2] G.Pancheri and Y. N. Srivastava, Eur. Phys. J. C 77, 150 (2017).

[3] M. M. Block, Phys. Rep. 436, 71 (2006).

[4] I. M. Dremin, Phys. Usp. 56, 3 (2013); J. Kašpar, V. Kundrát, M. Lokajíček, and J. Procházka, Nucl. Phys. B843, 84 (2011); R. Fiore, L. Jenkovszky, R. Orava, E. Predazzi, A. Prokudin, and O. Selyugin, Int. J. Mod. Phys. A 24, 2551 (2009).

[5] S. Donnachie, G. Dosch, P. V. Landshoff, and O. Natchmann, Pomeron Physics and $Q C D$ (Cambridge University Press, Cambridge, England, 2002).

[6] J. R. Forshaw and D. A. Ross, Quantum Chromodynamics and the Pomeron (Cambridge University Press, Cambridge, England, 1997); P. D. B. Collins, An Introduction to Regge Theory \& High Energy Physics (Cambridge University Press, Cambridge, England, 1977).
[7] D. A. Fagundes, M. J. Menon, and P. V. R. G. Silva, Int. J. Mod. Phys. A 32, 1750184 (2017).

[8] A. Donnachie and P. V. Landshoff, Phys. Lett. B 727, 500 (2013); Z. Phys. C 2, 55 (1979); 2, 372(E) (1979).

[9] P. C. Beggio and E. G. S. Luna, Nucl. Phys. A929, 230 (2014); D. A. Fagundes, E. G. S. Luna, M. J. Menon, and A. A. Natale, Nucl. Phys. A886, 48 (2012); E. G. S. Luna, Phys. Lett. B 641, 171 (2006); E. G. S. Luna, A. F. Martini, M. J. Menon, A. Mihara, and A. A. Natale, Phys. Rev. D 72, 034019 (2005); M. M. Block, E. M. Gregores, F. Halzen, and G. Pancheri, Phys. Rev. D 60, 054024 (1999).

[10] J. R. Cudell, V. V. Ezhela, P. Gauron, K. Kang, Yu. V. Kuyanov, S. B. Lugovsky, B. Nicolescu, and N.P. Tkachenko (COMPETE Collaboration), Phys. Rev. D 65, 074024 (2002).

[11] J. R. Cudell, V. V. Ezhela, P. Gauron, K. Kang, Yu. V. Kuyanov, S. B. Lugovsky, E. Martynov, B. Nicolescu, 
E. A. Razuvaev, and N. P. Tkachenko (COMPETE Collaboration), arXiv:hep-ph/0212101; B. Nicolescu, J. R. Cudell, V. V. Ezhela, P. Gauron, K. Kang, Yu. V. Kuyanov, S. B. Lugovsky, E. Martynov, E. A. Razuvaev, and N. P. Tkachenko (COMPETE Collaboration), Nucl. Phys. B, Proc. Suppl. 117, 400 (2003); J. R. Cudell, V. V. Ezhela, P. Gauron, K. Kang, Yu. V. Kuyanov, S. B. Lugovsky, E. Martynov, B. Nicolescu, E. A. Razuvaev, and N. P. Tkachenko (COMPETE Collaboration), Phys. Rev. Lett. 89, 201801 (2002).

[12] C. Patrignani et al. (Particle Data Group), Chin. Phys. C 40, 100001 (2016).

[13] G. Antchev et al. (TOTEM Collaboration), arXiv:1712.06153.

[14] G. Antchev et al. (TOTEM Collaboration), Report No. CERN-EP-2017-335.

[15] D. A. Fagundes, M. J. Menon, and P. V. R. G. Silva, Nucl. Phys. A966, 185 (2017).

[16] L. Lukaszuk and B. Nicolescu, Lett. Nuovo Cimento Soc. Ital. Fis. 8, 405 (1973).

[17] D. Joynson, E. Leader, B. Nicolescu, and C. Lopez, Nuovo Cim. A 30, 345 (1975).

[18] C. Ewerz, arXiv:hep-ph/0511196; arXiv:hep-ph/0306137; M. A. Braun, arXiv:hep-ph/9805394.

[19] R. Avila, P. Gauron, and B. Nicolescu, Eur. Phys. J. C 49, 581 (2007).

[20] E. Martynov and B. Nicolescu, Phys. Lett. B 778, 414 (2018).

[21] E. Martynov and B. Nicolescu, arXiv:1804.10139.

[22] V. A. Khoze, A. D. Martin, and M. G. Ryskin, Int. J. Mod. Phys. A 30, 1542004 (2015); Eur. Phys. J. C 74, 2756 (2014).
[23] V. A. Khoze, A. D. Martin, and M. G. Ryskin, Phys. Rev. D 97, 034019 (2018).

[24] V. A. Khoze, A. D. Martin, and M. G. Ryskin, Phys. Lett. B 780, 352 (2018).

[25] P. Lebiedowicz, O. Nachtmann, and A. Szczurek, Phys. Rev. D 98, 014001 (2018); E. Gotsman, E. Levin, and I. Potashnikova, arXiv:1807.06459; T. Csorgo, R. Pasechnik, and A. Ster, arXiv:1807.02897; V. A. Khoze, A. D. Martin, and M. G. Ryskin, Phys. Lett. B 784, 192 (2018); S. M. Troshin and N. E. Tyurin, arXiv:1805.05161; W. Broniowski, L. Jenkovszky, E. Ruiz Arriola, and I. Szanyi, arXiv:1806.04756.

[26] M. Broilo, E. G. S. Luna, and M. J. Menon, arXiv: 1803.06560.

[27] M. Broilo, E. G. S. Luna, and M. J. Menon, Phys. Lett. B 781, 616 (2018).

[28] R. F. Ávila and M. J. Menon, Nucl. Phys. A744, 249 (2004); J. B. Bronzan, G. L. Kane, and U. P. Sukhatme, Phys. Lett. B 49, 272 (1974).

[29] M. J. Menon and P. V. R. G. Silva, J. Phys. G 40, 125001 (2013); 41, 019501 (2014).

[30] M. M. Block and F. Halzen, Phys. Rev. D 86, 014006 (2012); 86, 051504 (2012).

[31] F. James, MINUIT Function Minimization and Error Analysis, Reference Manual, Version 94.1, CERN Program Library Long Writeup D506 (CERN, Geneva, 1998).

[32] P. R. Bevington and D. K. Robinson, Data Reduction and Error Analysis for the Physical Sciences (McGraw-Hill, New York, 1992).

[33] G. Grunberg and T. N. Truong, Phys. Rev. Lett. 31, 63 (1973). 